\title{
Radiological and Imaging Findings in Gastrointestinal Stromal Tumor: A Case Report
}

${ }^{1}$ Nilanshu Kataria, ${ }^{2}$ Vijinder Arora

\begin{abstract}
Introduction: Gastrointestinal stromal tumors (GISTs) are nonepithelial tumors arising from the interstitial cells of Cajal, which express KIT protein-CD117 on immunohistochemistry. It represents a wide clinical spectrum of tumors with different clinical presentations, locations, histology, and prognosis. Gastro intestinal stromal tumours (GISTs) can arise anywhere in the GIT, including the mesentery, omentum, and retroperitoneum. Because most of these tumors are submucosal in location, they usually attain a large size without causing bowel obstruction at the time of diagnosis. This report presents a case of GIST in a 30 -year-old male. The patient presented with acute abdomen without any symptoms of obstruction or perforation. He underwent ultrasonography in the radiology department which revealed a large mass along the small bowel with deposits along the mesentery and space occupying lesions (SOLs) in the liver. On contrast-enhanced computerised tomography (CECT), a possibility of GIST with deposits along the mesentery and liver was given, which was confirmed by histopathology.
\end{abstract}

Conclusion: Recognition and acquaintance with GISTs is primarily important for early detection which allows for more treatment options. These tumors are rare in people younger than 40 years of age, but can occur in people of any age.

Keywords: Contrast-enhanced CT, Gastrointestinal stromal tumor, Histopathology, Ultrasonography.

How to cite this article: Kataria N, Arora V. Radiological and Imaging Findings in Gastrointestinal Stromal Tumor: A Case Report. Curr Trends Diagn Treat 2018;2(2):112-114.

Source of support: Nil

Conflict of interest: None

\section{INTRODUCTION}

Gastrointestinal stromal tumors (GISTs) are the most common mesenchymal malignancy of the GI tract. ${ }^{1}$

Specific immunohistochemical properties of GISTs for KIT (CD117, a tyrosine kinase growth factor receptor) have allowed GISTs to be distinguished from other mesenchymal

\footnotetext{
${ }^{1}$ Junior Resident, ${ }^{2}$ Professor and Head

1,2Department of Radiodiagnosis and Imaging, Sri Guru Ram Das Institute of Medical Sciences \& Research, Amritsar, Punjab, India

Corresponding Author: Nilanshu Kataria, Department of Radiodiagnosis and Imaging, Sri Guru Ram Das Institute of Medical Sciences \& Research, Amritsar, Punjab, India, Phone: 8427449797, e-mail: nilanshukataria@gmail.com
}

tumors of the GI tract, such as leiomyomas, leiomyosarcomas, schwannomas, and neurofibromas. ${ }^{2}$

\section{CASE REPORT}

This is a report of a recent case of GIST of small intestinal origin. A 30-year-old male patient presented in the emergency department with complaints of acute abdomen and generalized tenderness over the abdomen. There were no complaints of constipation or diarrhea. On examination, the patient had marked guarding of the abdomen and tenderness to palpation. A myriad of provisional diagnoses for acute abdomen were considered.

The patient was referred to the Department of Radiodiagnosis for an X-ray of the abdomen erect and an ultrasound of the abdomen. No significant abnormality was detected at X-ray abdomen erect.

On ultrasonography of the abdomen, a large mass of an approximate size of $10 \times 8 \mathrm{~cm}$ was visualized in relation to small bowel in the mid abdomen (Fig. 1).

Few hypoechoic space-occupying lesions were seen in the liver (Fig. 2).

Few deposits were seen scattered along the small bowel mesentery (Fig. 3).

The patient was then advised to take the contrastenhanced CT (CECT) scan of the abdomen.

On CECT, there was a large exophytic heterogeneously enhancing soft tissue density lesion with large central cavitation and air fluid level noted along the antimesenteric border of the distal third and fourth parts of duodenum and proximal jejunum (Figs 4 and 5).

The arterial supply was found to be predominantly from superior mesenteric artery (SMA).

No evidence of any bowel obstruction was noted.

Multiple heterogeneously enhancing nodular omental and mesenteric deposits were seen, the largest of them in the right lumbar region with central areas of necrosis (Fig. 6).

Marked fat stranding was seen in the omentum.

Mild ascites was seen.

Few prominent sized lymph nodes were seen in the mesentery and para-aortic region, which was an unusual finding.

Few (at least 2) peripherally enhancing hypodense lesions were seen in segment VI of the liver (Figs 7 and 8).

On histopathological analysis, the tumor was confirmed to be GIST (Fig. 9). 


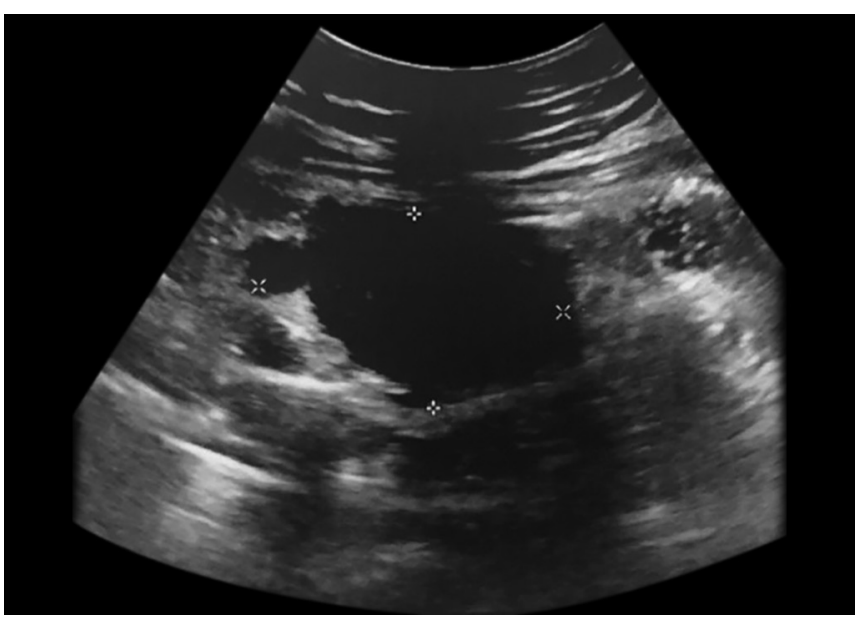

Fig. 1: Ultrasonography of abdomen shows a large mass of approximate size $10 \times 8 \mathrm{~cm}$ in relation to small bowel in the mid abdomen

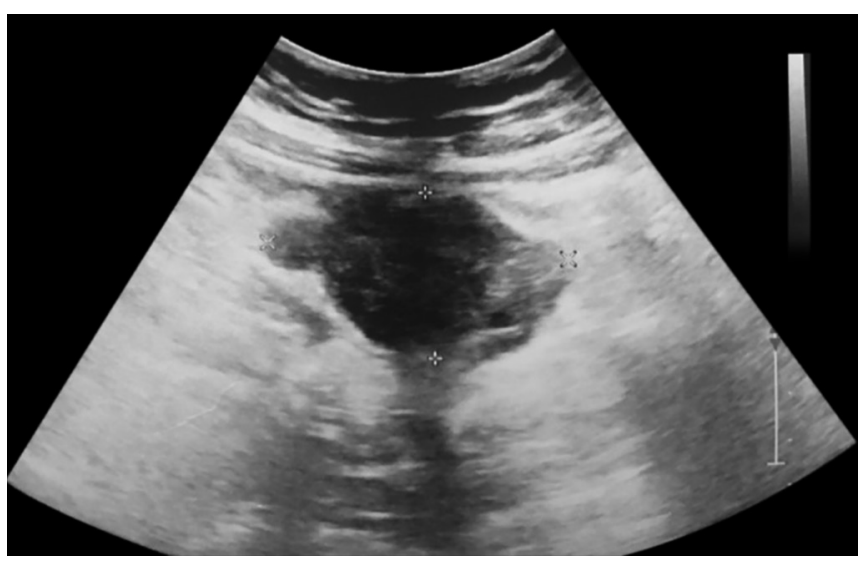

Fig. 3: Ultrasonography of abdomen shows a few deposits scattered along the small bowel mesentery

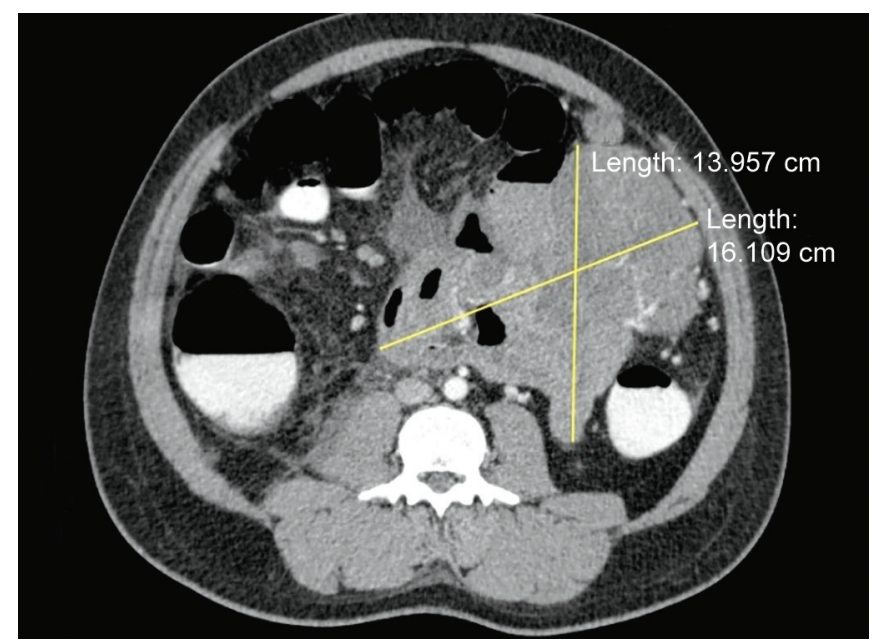

Fig. 5: On CECT of abdomen, the mass shows heterogeneous enhancement

The spindle cells were found to be immunoreactive to CD117 (c-kit)/DOG1/CD34/smooth muscle actin and immunonegative to $\mathrm{S} 100$ protein.

\section{DISCUSSION}

Up to $94 \%$ of patients with GISTs express CD117 (KIT protein) and, thus, share similar markers with the interstitial cells of Cajal. ${ }^{3}$

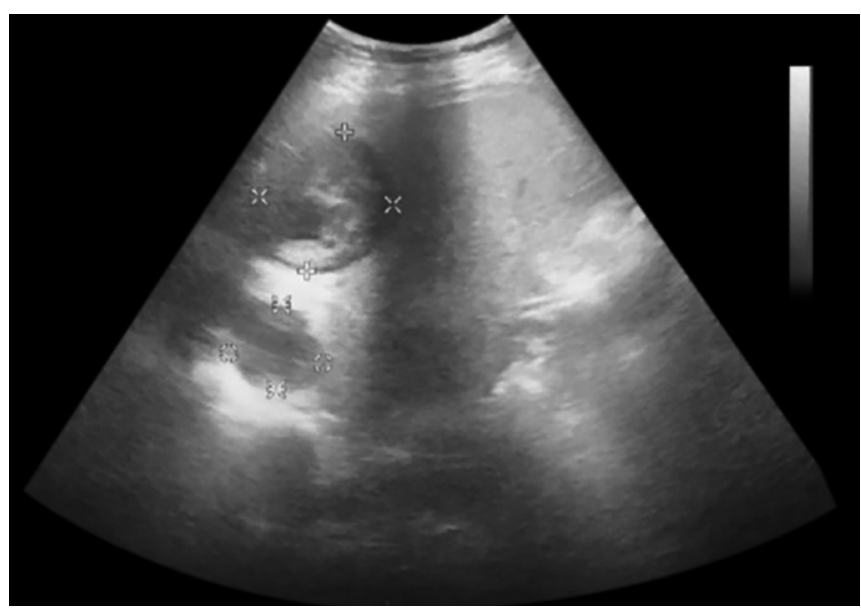

Fig. 2: Ultrasonography of abdomen shows hypoechoic space occupying lesions in the liver

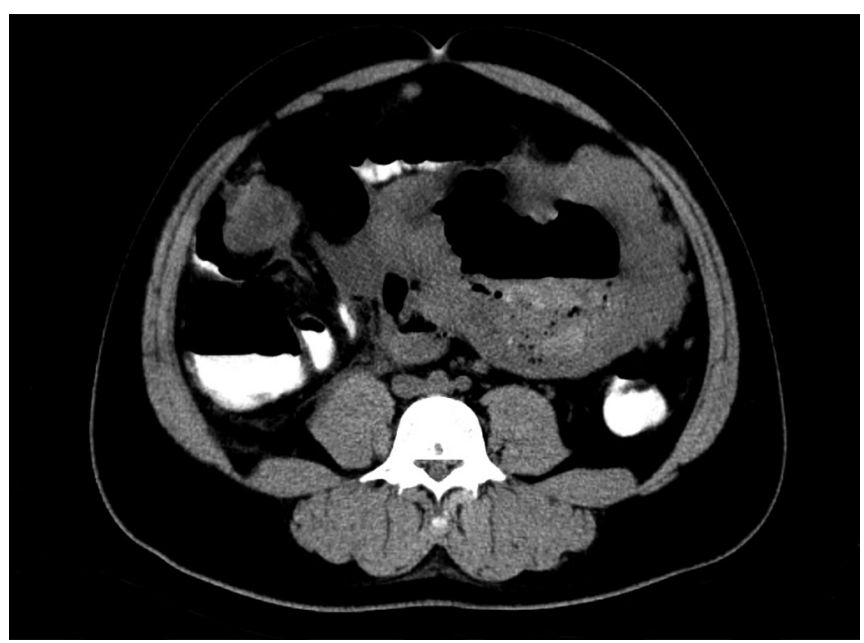

Fig. 4: On NECT of abdomen, a large exophytic mass is visualized with central cavitation and air-fluid level along the antimesenteric border of small bowel

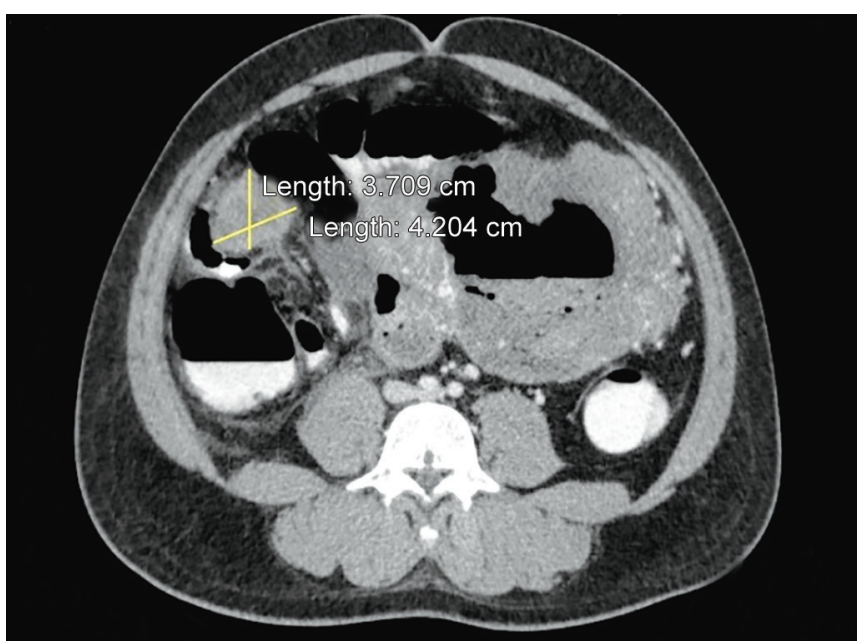

Fig. 6: CECT also reveals multiple heterogeneously enhancing nodular omental and mesenteric deposits, with central areas of necrosis

Mutations in the KIT oncogene of GIST resulted in constitutive activation of KIT receptor tyrosine kinase in GISTs. Thus, KIT is not only the principal marker for diagnostic purposes, but also a specific target for systemic therapy. ${ }^{4}$ 


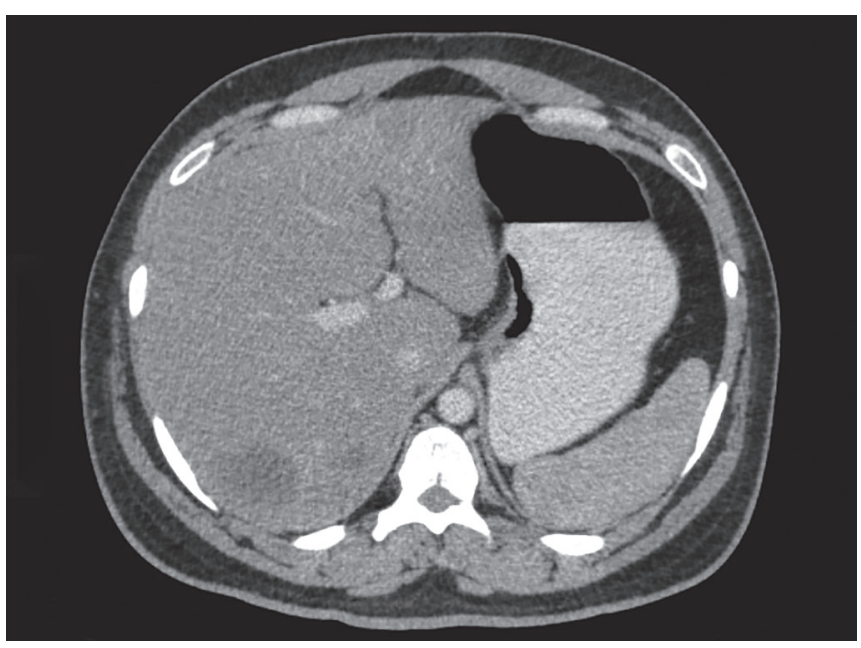

Fig. 7: NECT reveals two hypodense lesions in segment $\mathrm{VI}$ of liver

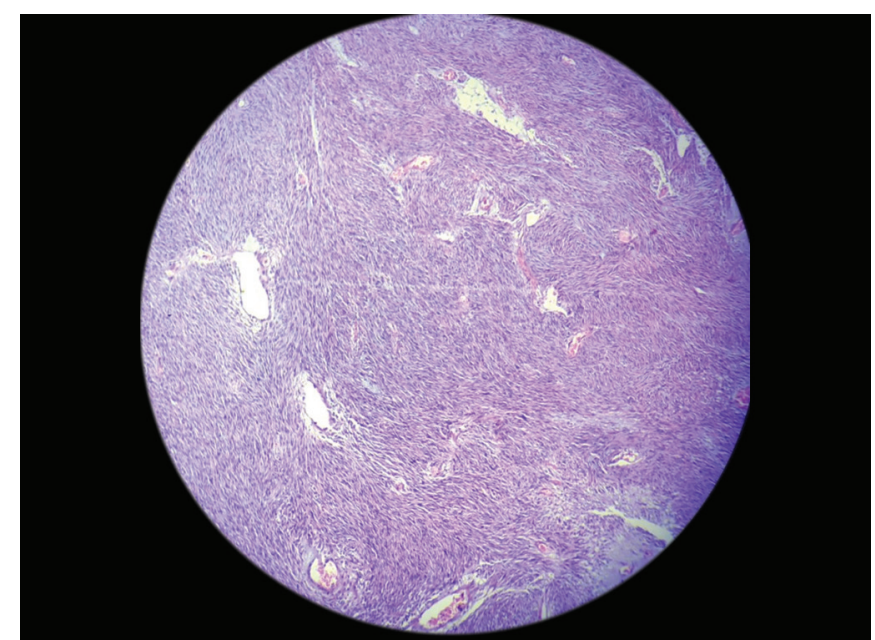

Fig. 9: Histopathological analysis shows multiple spindle cells as seen in gastrointestinal stromal tumor

On CT and magnetic resonance imaging (MRI), this tumor appears as a large heterogeneous mass with areas of low attenuation from hemorrhage, necrosis, or cyst formation and often grows in an exoenteric pattern with a large extraluminal component. ${ }^{5}$

Severe hemorrhage within the mass, which is associated with hypervascular stromal tumors, produces a fluid-fluid level. Calcification is occasionally demonstrated. ${ }^{6}$

Even in the presence of a large tumor, the incidence of bowel obstruction is rare. Some large cavitary tumors may show aneurysmal dilatation of the lumen, which is regarded as a fairly specific finding of lymphoma. ${ }^{7}$

Lymphadenopathy is not a common finding.

Metastasis occurs by direct extension and the hematogenous route. However, intraperitoneal spread (peritoneal leiomyosarcomatosis) may occur by intraperitoneal seeding via ascitic fluid or by embolic metastases or rupture of the

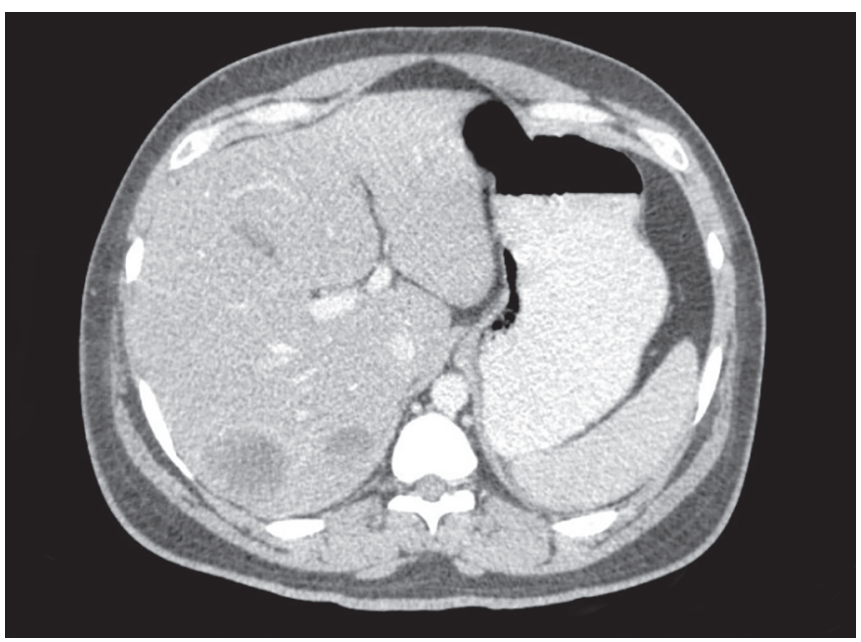

Fig. 8: CECT shows peripheral enhancement of the liver lesions

primary tumor and it is more commonly seen in significantly larger or exophytically growing tumors. ${ }^{8}$

The most common CT manifestations of peritoneal tumor seeding include multiple well-defined peritoneal nodules or masses with or without a smudged or granular pattern of omental infiltration. ${ }^{8}$

Lymphadenopathy is rare, and ascites may be seen but usually in a small amount. These CT findings may simulate those of peritoneal carcinomatosis from GI or ovarian malignancies. ${ }^{8}$

\section{REFERENCES}

1. George S, Desai J. Management of gastrointestinal stromal tumors in the era of tyrosine kinase inhibitors. Curr Treat Options Oncol 2002;3:489-496.

2. Miettinen M, Virolainen M, Maarit Sarlomo R. Gastrointestinal stromal tumors-value of CD34 antigen in their identification and separation from true leiomyomas and schwannomas. Am J Surg Pathol 1995;19:207-216.

3. Miettinen M, Lasota J. Gastrointestinal stromal tumorsdefinition, clinical, histological, immunohistochemical, and molecular genetic features and differential diagnosis. Virchows Arch 2001;438:1-12.

4. Hirota S, Isozaki K, Moriyama Y, et al. Gain-of-function mutations of c-kit in human gastrointestinal stromal tumors. Science 1998;279:577-580.

5. Levy AD, Remotti HE, Thompson WM, et al. Gastrointestinal stromal tumors: radiologic features with pathologic correlation. Radiographics 2003;23:283-304.

6. Chun HJ, Byun JY, Chun KA, et al. Gastrointestinal leiomyoma and leiomyosarcoma: CT differentiation. J Comput Assist Tomogr 1998;22:69-74.

7. Sandrasegaran K, Rajesh A, Rushing DA, et al. Gastrointestinal stromal tumors: CT and MRI findings. Eur Radiol 2005;15: 1407-1414.

8. Rha SE, Ha HK, Kim AY, et al. Peritoneal leiomyosarcomatosis originating from gastrointestinal leiomyosarcomas: CT features. Radiology 2003;227:385-390. 